\title{
PERFIS DE PERSONALIDADE NO PROGRAMA DE EDUCAÇÃO TUTORIAL EM ENGENHARIA CIVIL DA UFPR
}

Cibelle Renata do Prado - cibellerprado@gmail.com

Universidade Federal do Paraná

Av. Cel Francisco H. Dos Santos, 100 - Jardim das Américas

81531-980 - Curitiba - Paraná

Elvidio Gavassoni Neto-gavassoni@ufpr.br

Universidade Federal do Paraná

Av. Cel Francisco H. Dos Santos, 100 - Jardim das Américas

81531-980 - Curitiba - Paraná

Leonardo Meiguel-meiguelleonardo@gmail.com

Universidade Federal do Paraná

Av. Cel Francisco H. Dos Santos, 100 - Jardim das Américas

81531-980 - Curitiba - Paraná

Resumo: Em um mundo em que o autoconhecimento se torna importante para o bom desenvolvimento profissional e pessoal, é necessário aplicar métodos que auxiliam o crescimento pessoal e o desenvolvimento das organizações. Uma dessas ferramentas é o entendimento de perfis de personalidade dentro de grupos de trabalho. O programa de Educação Tutorial (PET) é um programa de educação pioneiro instituído pelo MEC que procura integrar ações inovadoras de ensino com ações de pesquisa e extensão. O PET Engenharia Civil da UFPR tem 36 anos de existência e se caracteriza por ser um grupo de trabalho colaborativo e horizontal sob orientação de um professor tutor. Com o propósito de identificar as personalidades do grupo PET Engenharia Civil a partir de 2015, foi realizadoo Teste MBTI (Myers-Briggs Type Indicator) em 30 integrantes atuais e egressos do programa. Quanto às características, segundo a metodologia MBTI, o resultado mostra a predominância do grupo sentinela, seguido de diplomatas, mas mostra também uma diversidade de perfis. Quanto às personalidades, observa-se o maior número de integrantes sendo defensores e em segundo um equilíbrio entre advogado e mediador. Tais características e personalidades são consistentes com as diretrizes do programa, como respeito à diversidade e ideais, ser horizontal, comprometimento e excelência em planejamento e execução de projetos.

Palavras-chave: Myers-Briggs Type Indicator. Autoconhecimento. Gestão de Pessoas.

\section{INTRODUÇÃO}

Nos últimos anos o estudo sobre os diferentes tipos de personalidade e seu impacto no desempenho de equipes de trabalho têm sido cada vez mais relevantes (MIN; SU, 2020). Tendo em vista essa ideia, buscando o melhor desenvolvimento de algumas empresas e organizações, pode-se utilizar a identificação e estudo da personalidade dos integrantes das equipes de trabalho para inseri-los em posições mais adequadas nas forças de trabalho. Sendo assim, o estudo dos perfis de personalidade se constitui de uma ferramenta de boa gestão de pessoas e de trabalho em equipes, principalmente nos impactos produzidos pela pandemia do COVID 19 (Di Fabio, Cheung e Peiró, 2020). 
Um exemplo do uso dos perfis de personalidade na gestão do trabalho em grupo é o estudo realizado com a Força Aérea dos Estados Unidos da América (DEVLIN; SINGH, 2010). O resultado da aplicação de testes de personalidade revela que os perfis de oficiais possuíam características em comum, sendo mais críticos e extrovertidos, já os membros alistados eram mais introvertidos, revelando assim possíveis ligações entre os perfis de personalidade e os cargos ocupados (DEVLIN; SINGH, 2010). Outro estudo de caso realizado por Hewett e Martini (2018) demonstra, aplicando o teste MBTI a uma amostra de instrutores de redação por ensino remoto, haver uma relação entre a dimensão mente (extroversão/introversão) e a preferência pelo ensino remoto. Outras aplicações revelam a relação entre os perfis de personalidade e o empoderamento entre indivíduos da sociedade (Greasley, Bocârnea, 2014); entre as habilidades de candidatos a empregos na indústria e suas características pessoais (Lee e Ahn, 2020) e entre os perfis de aprendizado de estudantes universitários (DUNGEY, YIELDER, 2017). Tais resultados demonstram que o estudo de perfis de personalidade pode ser fundamental para a gestão de pessoas em empresas e organizações, e os testes de personalidade são uma ferramenta adequada para esse estudo.

Com base nessas observações, o grupo PET Engenharia Civil da UFPR resolveu traçar o perfil de seus membros atuais e egressos a partir do ano de 2015, com o intuito de investigar os perfis de personalidade atraídos pelas atividades de educação tutorial. Além disso, seria possível fornecer ao departamento de gestão de pessoas do PET Engenharia Civil da UFPR uma ferramenta para seleção de novos integrantes e para gestão das relações entre os membros da equipe de trabalho do grupo. A identificação dos perfis de personalidade foi feita tendo por base um estudo de duas pesquisadoras, Isabel Briggs Myers e Katharine Briggs. O estudo ficou conhecido como Teste MBTI (Myers-Briggs Type Indicator), nele Myers e Briggs utilizaram também a teoria de Carl Jung descrita no livro "Tipos Psicológicos" (1921).

O PET Engenharia Civil tem mais de 36 anos de história (PET CIVIL, 2009), apesar disso, foi utilizado como amostra os membros dos últimos 5 anos. $\mathrm{O}$ grupo tem basicamente como missão promover a melhoria do curso de Engenharia Civil por meio de ações inovadoras de ensino, contribuir para o bem-estar da sociedade, de forma técnica e humana, articulando ensino, pesquisa e promover projetos ou atividades de extensão. Além disso o trabalho do grupo se baseia na horizontalidade e no trabalho colaborativo, ou seja, não há níveis de hierarquia e todos possuem mesmo poder decisório e as diferentes atividades e responsabilidades são compartilhadas entre todos os integrantes. Com tais características, nota-se que seus membros devem possuir respeito à diversidade, empatia entre os mesmos, resiliência diante situações de tensão e criatividade, além de possuírem comprometimento e serem leais aos colegas do grupo e aos princípios do programa de educação tutorial (MARTIN, 2005).

\section{METODOLOGIA}

A identificação dos perfis de personalidade utilizou o questionário baseado no trabalho de Myers-Briggs, inserido no site 16Personalities (2020), que usa o método NERIS Type Explorer e difere-se por conter um traço de personalidade a mais (-A/-T). Além disso, por não julgar conceitos junguianos, ele avalia cinco aspectos independentes: mente, energia, natureza, tática e identidade.

Um e-mail com instruções sobre o teste foi enviado para os 47 integrantes e ex-integrantes do grupo PET Engenharia Civil UFPR selecionados a partir de 2015.

Dos 30 participantes 4 pessoas já se formaram na instituição (13,33\%), 12 estudam Engenharia Civil e não fazem mais parte do PET (40\%) e 14 ainda participam ativamente do programa (46,67\%), sendo 13 estudantes de Engenharia Civil e 1 o tutor do grupo. Com base nas respostas, ao todo são 21 homens $(70 \%)$ e 9 mulheres (30\%). 
As combinações existentes no teste são diversas, totalizando 16 personalidades diferentes. Os resultados consistem em uma combinação de 4 letras, uma para cada dimensão, dentre 8 possibilidades ( 2 para cada dimensão), que definem uma classificação objetiva de cada tipo de personalidade. $\mathrm{O}$ teste possui 4 dimensões que definem uma personalidade (16Personalidades, 2020), são elas abaixo:

1- Mente: fonte de energia

Extrovertido (E): Tende a focar no mundo exterior e direcionar a sua atenção para pessoas ou objetos, se sente energizado por meio de outras pessoas, as vezes pode agir antes de pensar.

Introvertido (I): Atende a realidade interior, se concentra em conceitos e ideias, se sente engajado por atividades solitárias e antes de agir pensa muito.

2- Energia: modo de perceber o mundo

Sensoriais (S): Pessoa que basicamente usa os cinco sentidos para interpretar fatos ou eventos, consciência voltada para o que mais existe de concreto, realidade.

Intuitivo $(\mathrm{N})$ : Confia mais em sua intuição para atribuir significado, consciência voltada para o abstrato, ao intangível, ao lado simbólico.

3- Natureza: maneira de avaliação, julgamento, organização e decisão

Racionalistas (T): Confia suas decisões em coisas concretas, baseia-se em objetivos, raciocínio lógico, atua de forma clara e objetiva.

Sentimentais (F): Toma decisões levando em consideração seus valores e sentimentos internos e externos.

4- Tática: estilo de vida

Julgadores (J): Prefere seguir regras claras e objetivas, vive melhor de maneira mais planejada, e se sente melhor quando toma decisões.

Perceptivos (P): Aprecia a liberdade, usa improvisações, prefere uma vida com maior flexibilidade, gosta de adaptar-se, se sente melhor quando apresenta opções em aberto.

Essas oito características geram 16 tipos possíveis de personalidades segundo o método MBTI. Os 16 perfis de personalidades, são então agrupados em 4 grupos: analistas, diplomatas, sentinelas e exploradores (Quadro 1). Os perfis inclusos em cada grupo compartilham características similares.

\begin{tabular}{|c|c|c|c|}
\hline Analista & Diplomata & Sentinela & Explorador \\
\hline Arquiteto (INTJ) & Advogado (INFJ) & Logístico (ISTJ) & Virtuoso (ISTP) \\
\hline Lógico (INTP) & Mediador (INFP) & Defensor (ISFJ) & Aventureiro (ISFP) \\
\hline Comandante (ENTJ) & Protagonista (ENFJ) & Executivo (ESTJ) & Empresário (ESTP) \\
\hline Inovador (ENTP) & Ativista (ENFP) & Cônsul (ESFJ) & Animador (ESFP) \\
\hline
\end{tabular}

Uma quinta dimensão, característica do teste 16Personalidades (2020), não interfere nas personalidades apresentadas no Quadro 1, sendo apenas mais um traço individual.

5- Identidade: modo de tomar decisões

Assertivos (A): Confiante, não deixa o nervosismo atrapalhar seus objetivos. Se sente mais seguro de suas habilidades para lidar com dificuldades e coisas inesperadas.

Cautelosos (T): Motivado pelo sucesso, tende a ser perfeccionista e ansioso, mas ao mesmo 
"Os desafios para formar hoje o engenheiro do amanhã"

tempo cuidadoso e atencioso, estando sempre em busca de melhoria.

\section{RESULTADOS}

Dos 47 e-mails enviados houveram 30 respostas, uma taxa de retorno de 63,83\%. O gráfico 1 mostra a distribuição de cada tipo de personalidade dos 30 participantes do estudo, esses são os principais dados da pesquisa, pois mostram que o PET Engenharia Civil UFPR é um grupo diversificado, apresentado ao menos um indivíduo para 11 diferentes perfis de personalidade. Contudo, observa-se que há uma maior porcentagem do tipo ISFJ (defensor) e que 5 dos 16 traços de personalidade não estão presentes na amostra: ENTJ, ENTP, ISTJ, ISTP e ESTP.

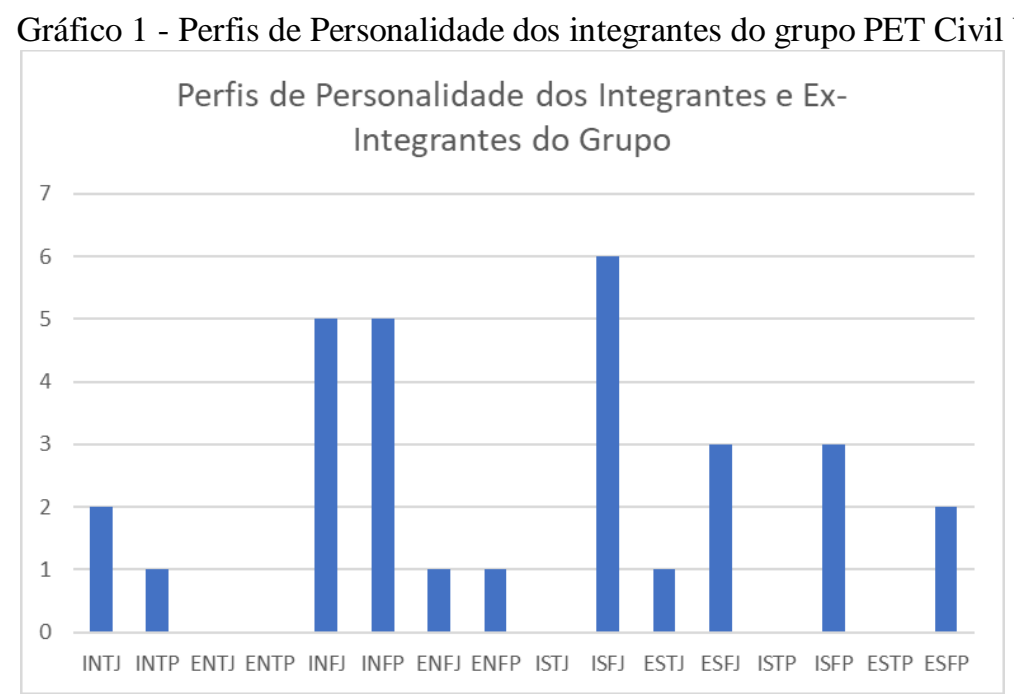

Fonte: Os Autores.

Ao comparar homens e mulheres, observa-se que os homens possuem tipos de personalidade mais variados, compreendendo os quatro grandes grupos de perfis, as personalidades do grupo sentinela são as com maior destaque e a personalidade defensor (ISFJ) com a maior quantidade de pessoas, $23,81 \%$.

Já dentre as mulheres há uma concentração maior no grupo diplomata $(66,67 \%)$ e sentinela $(33,33 \%)$, com destaque nas personalidades advogado (INFJ), mediador (INFP) e cônsul (ESFJ).

Gráfico 2 - Perfis de Personalidade dos homens e mulheres integrantes e ex-integrantes do grupo.

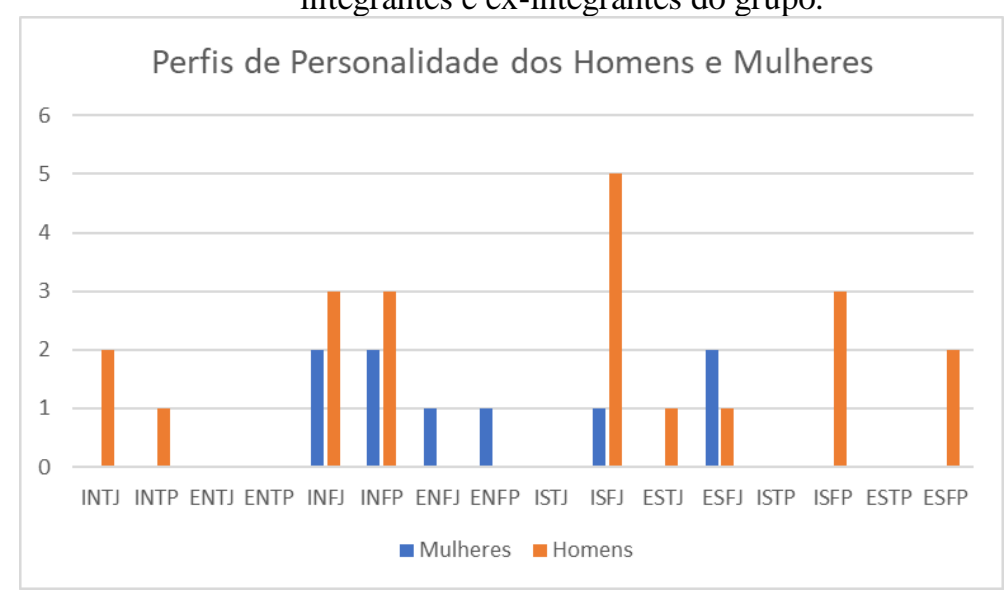

Fonte: Os Autores. 
A análise individual das personalidades revela que o grupo aparenta ser bastante heterogêneo, por possuir 11 das 16 disponíveis. Mas, quando separadas nos quatro grupos de personalidade, observa-se um determinado padrão. O quadro 2 mostra que em 2015, 2016, 2018 e 2020 a maior parte dos admitidos no processo seletivo fazem parte do grupo sentinela, já nos anos de 2016 e 2019 o predomínio foi do grupo diplomata. O recorte anual se justifica pois o grupo se renova anualmente em função de seus processos seletivos semestrais e alta rotatividade de integrantes.

O grupo diplomata é caracterizado pela presença mútua dos traços intuitivo (N) e sentimental (F), assim, é tipicamente associado à empatia, habilidades diplomáticas e cooperação. No entanto, essa personalidade pode ter problemas em tomar decisões difíceis.

Já o sentinela possui os traços observador $(\mathrm{S})$ e julgador $(\mathrm{J})$, tendo a ordem, segurança e estabilidade como principais atributos. Também tendem a ser trabalhadores, meticulosos e tradicionais, podendo ter problemas ao aceitar diferentes pontos de vista.

Quadro 2 - Grupo dominante de características de acordo com $o$ ano de ingresso no PET Engenharia Civil UFPR.

\begin{tabular}{|c|c|c|c|c|}
\hline Ano & Tipo & Quantidade & Grupo & Grupo dominante \\
\hline 2015 & $\begin{array}{l}\text { INFP } \\
\text { ISFJ } \\
\text { ESFJ } \\
\text { ISFP }\end{array}$ & $\begin{array}{l}1 \\
1 \\
1 \\
1\end{array}$ & $\begin{array}{c}\text { Diplomata } \\
\text { Sentinela } \\
\text { Sentinela } \\
\text { Explorador }\end{array}$ & Sentinela \\
\hline 2016 & $\begin{array}{l}\text { INTJ } \\
\text { INFJ } \\
\text { INFP }\end{array}$ & $\begin{array}{l}1 \\
1 \\
1\end{array}$ & $\begin{array}{l}\text { Analista } \\
\text { Diplomata } \\
\text { Diplomata }\end{array}$ & Diplomata \\
\hline 2017 & $\begin{array}{l}\text { INTJ } \\
\text { INFJ } \\
\text { ISFJ } \\
\text { ESFJ }\end{array}$ & $\begin{array}{l}1 \\
1 \\
1 \\
1\end{array}$ & $\begin{array}{c}\text { Analista } \\
\text { Diplomata } \\
\text { Sentinela } \\
\text { Sentinela }\end{array}$ & Sentinela \\
\hline 2018 & $\begin{array}{l}\text { INFP } \\
\text { INTP } \\
\text { ENFJ } \\
\text { ENFP } \\
\text { ISFJ } \\
\text { ESFJ }\end{array}$ & $\begin{array}{l}1 \\
1 \\
1 \\
1 \\
3 \\
1\end{array}$ & $\begin{array}{c}\text { Diplomata } \\
\text { Analista } \\
\text { Diplomata } \\
\text { Diplomata } \\
\text { Sentinela } \\
\text { Sentinela }\end{array}$ & Sentinela \\
\hline 2019 & $\begin{array}{l}\text { INFJ } \\
\text { INFP } \\
\text { ISFP } \\
\text { ESFP }\end{array}$ & $\begin{array}{l}2 \\
2 \\
2 \\
1\end{array}$ & $\begin{array}{l}\text { Diplomata } \\
\text { Diplomata } \\
\text { Explorador } \\
\text { Explorador }\end{array}$ & Diplomata \\
\hline 2020 & $\begin{array}{l}\text { INFJ } \\
\text { ISFJ } \\
\text { ESTJ } \\
\text { ESFP }\end{array}$ & $\begin{array}{l}1 \\
1 \\
1 \\
1\end{array}$ & $\begin{array}{l}\text { Diplomata } \\
\text { Sentinela } \\
\text { Sentinela } \\
\text { Explorador }\end{array}$ & Sentinela \\
\hline
\end{tabular}

Fonte: Os Autores.

Observando cada característica de personalidade separadamente é possível identificar as que o grupo mais possui em comum.

O gráfico 3 mostra que entre as características extrovertido (E) e introvertido (I), 73\% dos petianos apresentam a característica introvertido. Segundo o trabalho de Akroyd, Bernold e O'Brien (1998), o estudo entre 83 estudantes de engenharia da Universidade da Carolina do 
Norte indicou que 59\% dos participantes também possuem a característica I como predominante, revelando que tal característica possa pertencer ao grupo mais amplo de estudantes de engenharia e não propriamente ao programa de educação tutorial.

Gráfico 3 - Proporção das características da mente E e I.

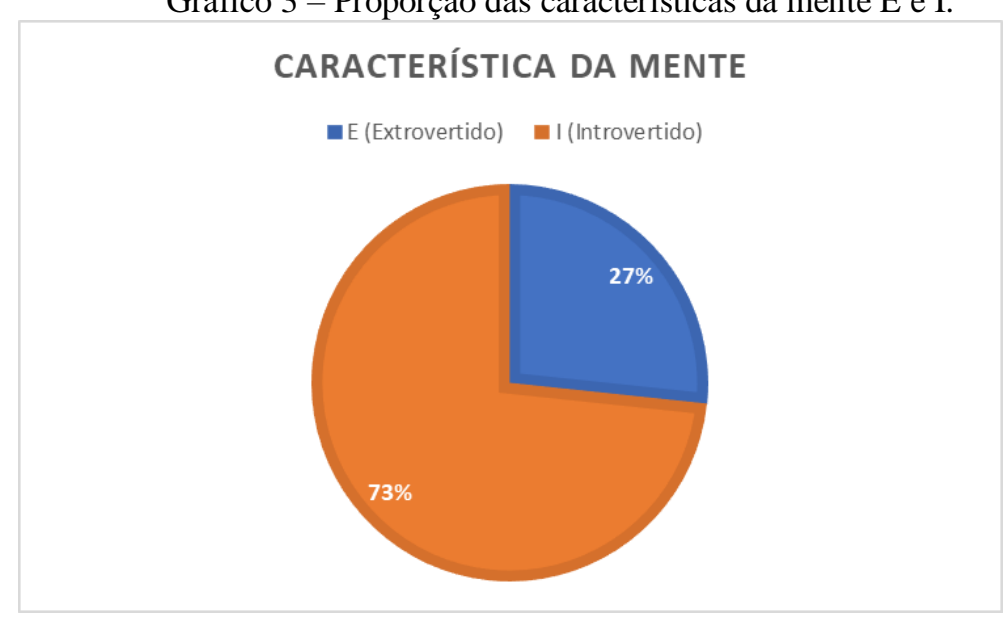

Fonte: Os Autores.

Além da característica de energia mostrar equilíbrio (gráfico 4), ela é interessante por ser o traço que define se uma pessoa tem a personalidade advogado (INFJ) ou defensor (ISFJ). Essas personalidades, como visto no Gráfico 1, são duas das três que mais aparecem entre os que responderam à pesquisa feita com integrantes do grupo PET Engenharia Civil UFPR. Assim, mesmo INFJ e ISFJ pertencerem à grupos distintos, diplomata e sentinela, é possível perceber que apenas um traço as difere e que a precisão do teste pode interferir nesse resultado. Os portadores dessas duas personalidades são $36,67 \%$ dos petianos.

Gráfico 4 - Proporção das características de energia S e N.

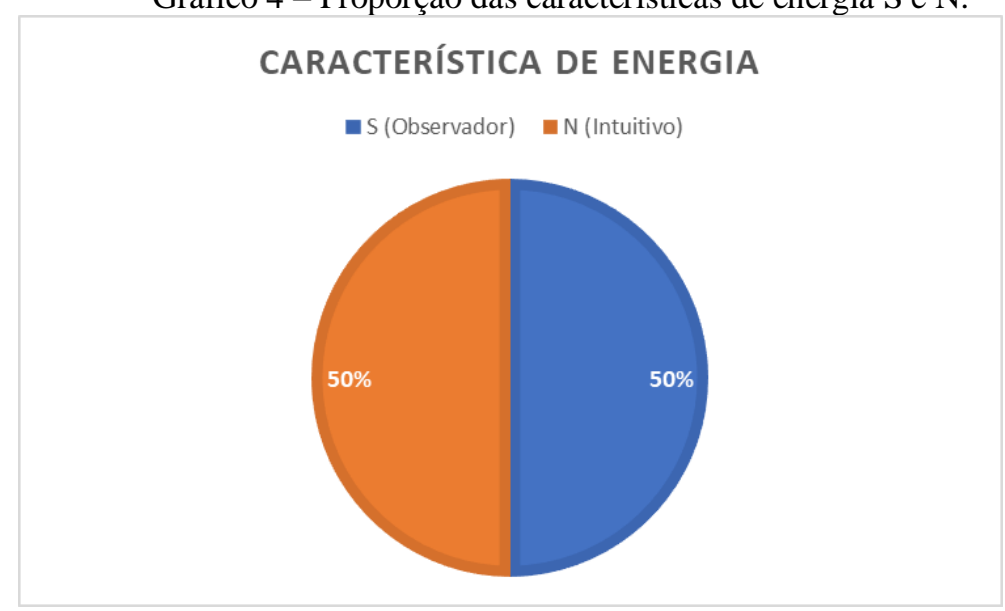

Fonte: Os Autores.

A característica de natureza é peculiar, visto que $87 \%$ dos integrantes (Gráfico 5) apresentaram-se como sentimental (F), enquanto o estudo de Akroyd, Bernold e O'Brien (1998) mostra que $75 \%$ dos estudantes de engenharia, na verdade, possuem o perfil racional, indicando que o programa de educação tutorial possa atrair um determinado grupo não muito majoritário entre os estudantes de engenharia. 
"Os desafios para formar hoje o engenheiro do amanhã"

Gráfico 5 - Proporção das características de natureza T e F.

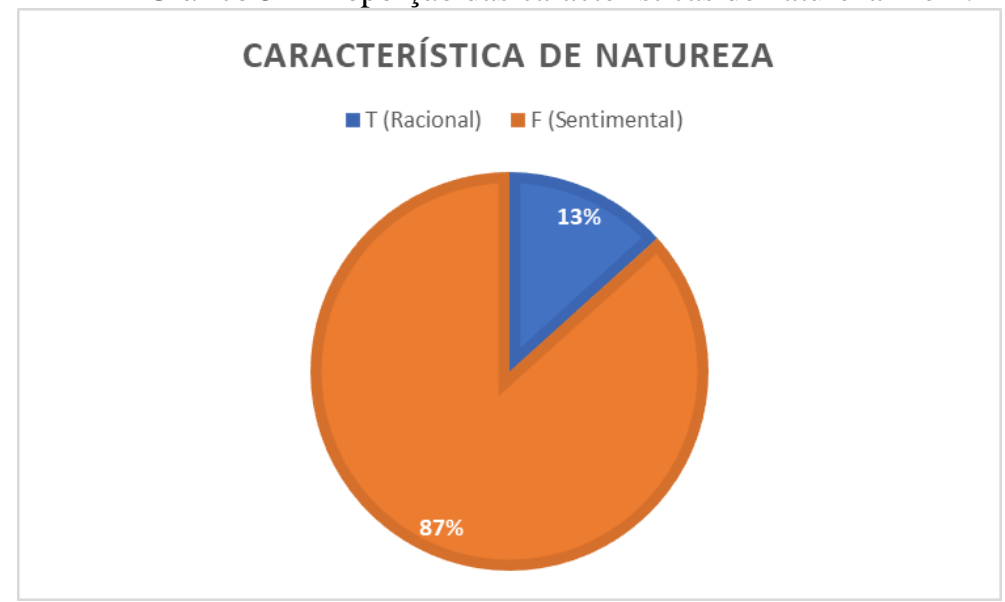

Fonte: Os Autores.

Segundo Devling e Singh (2010), as pessoas com características de explorador preferem flexibilidade e liberdade, além de se adaptarem melhor às mudanças de última hora. Esse traço estar presente em $40 \%$ dos integrantes da pesquisa (gráfico 6) pode ser justificado pelas diretrizes do próprio programa, em que se busca a divisão de tarefas. Assim, é relevante o suficiente para a inovação e adaptação, mas a característica julgador $(60 \%)$ é o que faz o grupo ser equilibrado e as decisões serem feitas respeitando possíveis limitações.

Gráfico 6 - Proporção das características de táticas J e P.

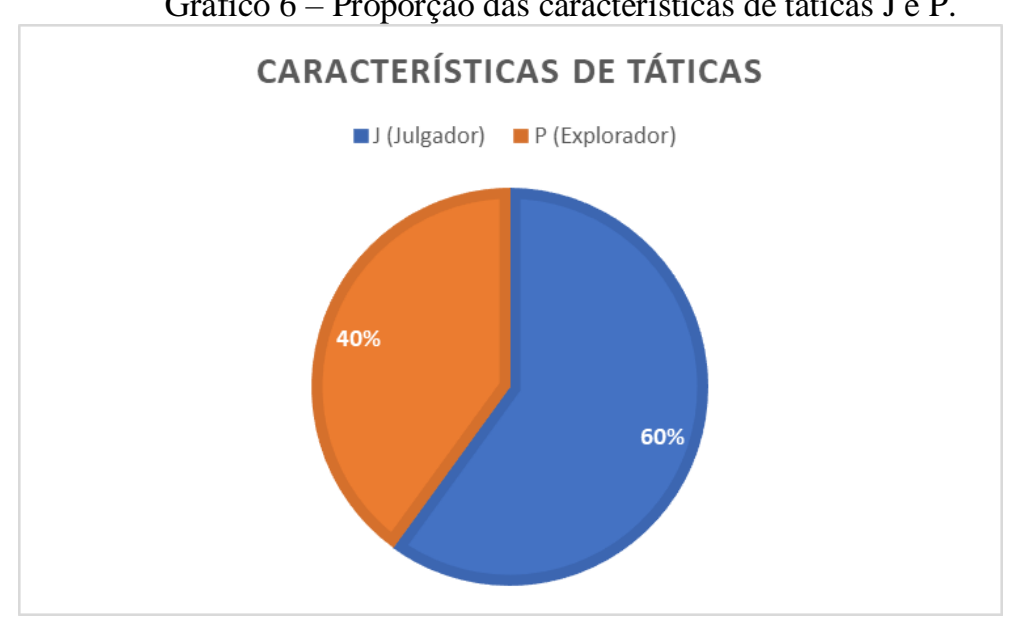

Fonte: Os Autores.

A última dimensão, que separa o grupo em duas partes, independente da personalidade que o indivíduo possui, teve com maior proporção entre os petianos a característica cauteloso (Gráfico 7). Esse traço pode ser percebido no dia a dia do grupo, pois os projetos que exigem atenção e horizontalidade fazem com que os integrantes do grupo precisem ser cuidadosos com as suas responsabilidades. Mas, a presença de uma grande porcentagem do traço assertivo justifica o grupo ser capaz de lidar com coisas inesperadas e difíceis, quando necessário. 


\section{COBENGE (C) COBENCE 2020 da ABENGE}

\section{"Os desafios para formar hoje o engenheiro do amanhã"}

Gráfico 7 - Proporção das características de identidade A e T.

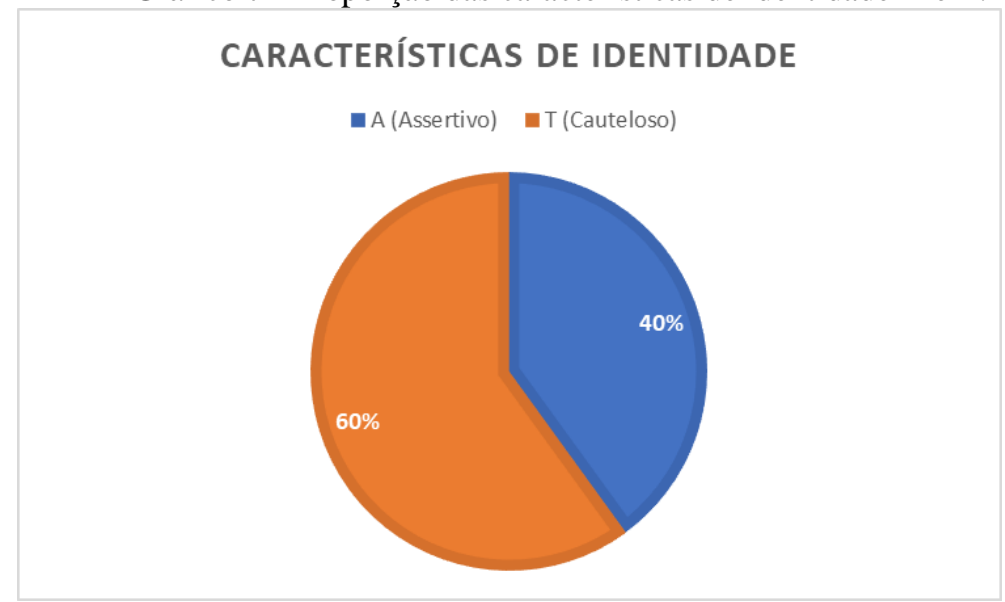

Fonte: Os Autores.

Quando feita a análise dos traços por gênero (Gráfico 8), percebe-se que as mulheres possuem em maior porcentagem o traço intuitivo $(66,67 \%)$ do que os homens $(42,85 \%)$. O mesmo ocorre na característica de tática, onde mulheres são cautelosas $(88,89 \%)$ e os homens assertivos $(52,38 \%)$. Os demais traços possuem dominância semelhante.

Se as características forem analisadas separadamente, a dominância dos traços seria de I, $\mathrm{S}, \mathrm{N}, \mathrm{F}, \mathrm{J}$ e $\mathrm{T}$, que formariam justamente as personalidades mais apresentadas pelo grupo: defensor (ISFJ) e advogado (INFJ), com 20 e 16,67\% respectivamente, junto ao traço cauteloso (T). Se a mesma análise for feita de acordo com o gênero, as mulheres formariam a personalidade advogado e cauteloso (INFJ-T) e a dominância de traços dos homens levaria a defensor assertivo (ISFJ-A). Já uma análise feita por ano (Quadro 3) reafirma o padrão observado no Quadro 2, com diferença no ano de 2015, em que traços do grupo explorador aparecem.

Gráfico 8 - Traços de personalidade dos integrantes e ex-integrantes do PET Civil UFPR de acordo com o gênero.

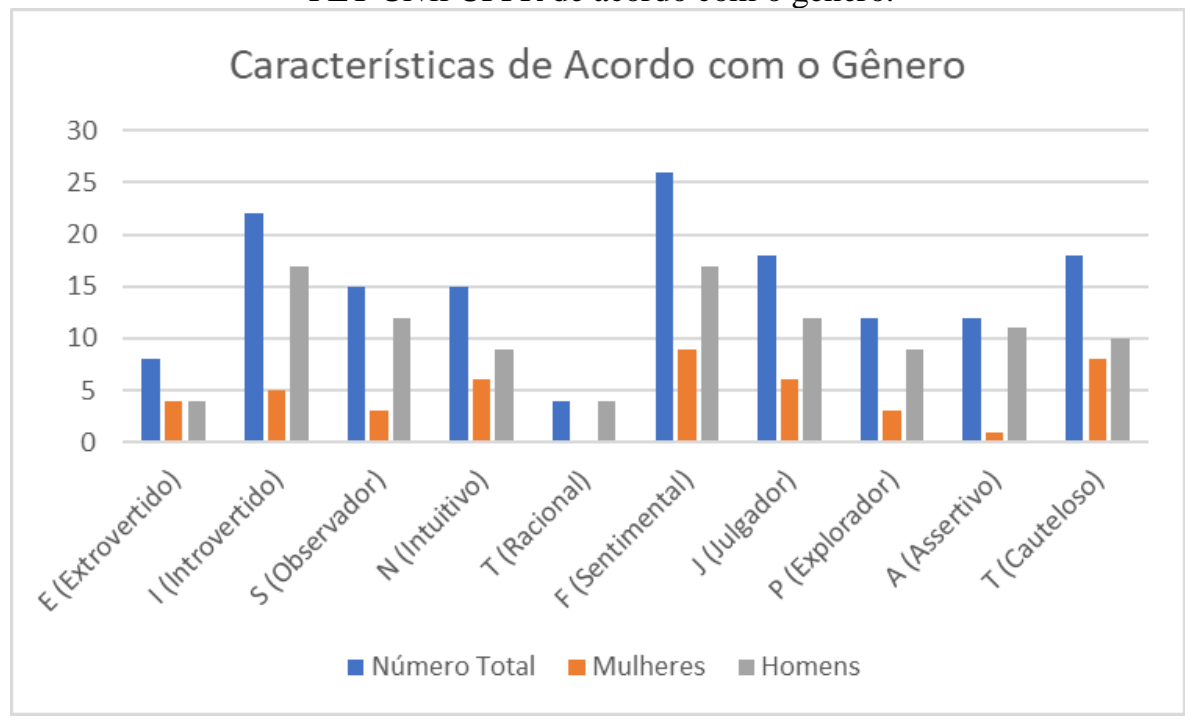

Fonte: Os Autores. 
Quadro 3 - Traços dominantes do grupo de 2015 a 2020

\begin{tabular}{|c|c|c|c|}
\hline Ano & Traços dominantes & Personalidade & Grupo \\
\hline 2015 & I, S, F, P, T & Virtuoso & Explorador \\
\hline 2016 & I, N, F, J, A & Advogado & Diplomata \\
\hline 2017 & I, S, N, F, J, T, A & Defensor e Advogado & Sentinela e Diplomata \\
\hline 2018 & I, S, F, J, T & Defensor & Sentinela \\
\hline 2019 & I, N, F, P, T & Mediador & Diplomata \\
\hline 2020 & E, I, S, F, J, T, A & Defensor e Cônsul & Sentinela \\
\hline
\end{tabular}

Fonte: Os Autores.

\section{CONCLUSÃO}

A aplicação da metodologia MBTI revelou-se uma ferramenta importante na gestão de pessoas do grupo PET Engenharia Civil da UFPR. De acordo com o resultado da amostra pesquisada o grupo é heterogêneo, pois possui 11 das 16 personalidades diferentes. $\mathrm{O}$ grupo de personalidades predominante em quatro anos é o sentinela, seguido por dois anos do diplomata. A personalidade que mais aparece nos últimos 5 anos é a de defensor, já em segundo aparecem duas personalidades, a de advogado e mediador. Ambos grupos e personalidades vão de encontro com as diretrizes do programa, pois prezam a horizontalidade, respeitam as diversidades e demonstram responsabilidade e empenho no desenvolvimento e execução das atividades e dos projetos do grupo. Assim, os sentinelas tendem a ser indivíduos mais guardiões, vigias ou executores e, por outro lado, os diplomatas prezam pelos ideais e pelo respeito.

Outra análise realizada apresenta as características dentro das personalidades que mais aparecem no grupo, como introversão, observador, sentimental e julgador. Esse conjunto forma a personalidade de defensor que está dentro do grupo de sentinela. Sendo assim, percebe-se que o programa atrai pessoas com características e personalidades parecidas, que prezam pelo respeito, sendo indivíduos engajados em defender os ideais do grupo e planejar e executar da melhor forma os projetos que o grupo se envolve. Algumas dessas características são compartilhadas com a maioria dos alunos de graduação de engenharia, como o traço introvertido, e outras se destacaram por serem mais predominantes no grupo PET Engenharia Civil da UFPR, como no caso da característica sentimental.

\section{REFERÊNCIAS}

AKROYD, Duane; BERNOLD, Leonhard E.; O'BRIEN, Terrance P. Myers-Briggs type indicator and academic achievement in engineering education. International Journal of Engineering Education, Great Britain, v. 14, n. 5, p. 311-315, 1998.

CIVIL, Pet Engenharia. PET 25 anos: Resgate Histórico do PET Engenharia Civil da UFPR. Curitiba, 2008. $114 \mathrm{p}$.

DEVLIN, M. Scott; SINGH, Amarjit. MBTI Personality and Hemisphericity of a U.S. Air Force Group. Leadership And Management In Engineering, [S.L.], v. 10, n. 3, p. 108-120, jul. 2010. American Society of Civil Engineers (ASCE). http://dx.doi.org/10.1061/(asce)lm.1943-5630.0000063.

DI FABIO, Annamaria; CHEUNG, Fanny M.; PEIRÓ, José María. Editorial to special issue "Personality and individual differences and healthy organizations". Personality And Individual Differences, [S.L.], v. 166, p. 110196, nov. 2020. Elsevier BV. http://dx.doi.org/10.1016/j.paid.2020.110196.

DUNGEY, G.; YIELDER, J.. Student personality and learning styles: a comparison between radiation 
therapy and medical imaging undergraduate students in new zealand. Radiography, [S.L.], v. 23, n. 2, p. 107-111, maio 2017. Elsevier BV. http://dx.doi.org/10.1016/j.radi.2016.11.005.

GREASLEY, Paul E.; BOCÂRNEA, Mihai C.. The Relationship between Personality Type and the Servant Leadership Characteristic of Empowerment. Procedia - Social And Behavioral Sciences, [S.L.], v. 124, p. 11-19, mar. 2014. Elsevier BV. http://dx.doi.org/10.1016/j.sbspro.2014.02.454.

HEWETT, Beth L.; MARTINI, Rebecca Hallman. Educating Online Writing Instructors Using the Jungian Personality Types. Computers And Composition, [S.L.], v. 47, p. 34-58, mar. 2018. Elsevier BV. http://dx.doi.org/10.1016/j.compcom.2017.12.007.

LEE, Dongseop; AHN, Changkuk. Industrial human resource management optimization based on skills and characteristics. Computers \& Industrial Engineering, [S.L.], v. 144, p. 106463, jun. 2020. Elsevier BV. http://dx.doi.org/10.1016/j.cie.2020.106463.

MARTIN, Maria da Graça Moraes Braga. O Programa de Educação Tutorial-PET: Formação ampla na graduação. 2005. 95 f. Dissertação (Mestrado) - Setor de Educação, Universidade Federal do Paraná, Curitiba, 2005.

MIN, Hanyi; SU, Shiyang. Examining relationships between personality profiles and organizational health outcomes. Personality And Individual Differences, [S.L.], v. 164, p. 110118, out. 2020. Elsevier BV. http://dx.doi.org/10.1016/j.paid.2020.110118.

16PERSONALITIES. Our framework. Disponível em:

<https://www.16personalities.com/articles/our-theory>. Acesso em: 30 jul 2020.

\title{
PERSONALITY PROFILES IN THE TUTORIAL EDUCATION PROGRAM IN CIVIL ENGINEERING FROM UFPR
}

\begin{abstract}
In a world in which self-knowledge becomes important for good professional and personal development, it is necessary to apply methods that help personal growth and the development of organizations. One of these tools is the understanding of personality profiles within working groups. The Tutorial Education program (PET) is a pioneering education program instituted by MEC that seeks to integrate innovative teaching actions with research and extension actions. The PET Civil Engineering from UFPR is 36 years old and is characterized by being a collaborative and horizontal working group under the guidance of a tutor teacher. In order to identify the personality types of the PET Engenharia Civil group since 2015, the MBTI Test (Myers-Briggs Type Indicator) was carried out on 30 current members and ex-members of the program. About the characteristics, according to the MBTI methodology, the result shows the predominance of the sentinel group, followed by diplomats, but it also shows a diversity of profiles. About the personalities, there is a greater number of members being defenders and secondly a balance between advocate and mediator. Such characteristics and personalities are consistent with the program's guidelines, such as respect for diversity and ideals, being horizontal, commitment and excellence in project planning and execution.
\end{abstract}

Keywords: Myers-Briggs Type Indicator. Self knowledge. People management. 